\title{
Assessment of the predictive role of pretreatment Ki-67 and Ki-67 changes in breast cancer patients receiving neoadjuvant chemotherapy according to the molecular classification: a retrospective study of 1010 patients
}

\author{
Rui Chen ${ }^{1}$ - Yin Ye ${ }^{1}$. Chengcheng Yang ${ }^{1}$. Yang Peng ${ }^{1}$ - Beige Zong ${ }^{1}$ - Fanli Qu ${ }^{1}$ - Zhenrong Tang ${ }^{1}$ - Yihua Wang ${ }^{1}$. \\ Xinliang $\mathrm{Su}^{1} \cdot$ Hongyuan $\mathrm{Li}^{1}$. Guanglun Yang ${ }^{1}$. Shengchun $\mathrm{Liu}^{1}$
}

Received: 3 December 2017 / Accepted: 19 February 2018 / Published online: 26 February 2018

(c) The Author(s) 2018. This article is an open access publication

\begin{abstract}
Purpose To assess the predictive role of pretreatment ki67 and Ki67 changes in breast cancer (BC) patients treated with neoadjuvant chemotherapy (NAC) in various molecular subtypes.

Methods $1010 \mathrm{BC}$ patients who had undergone anthracycline and taxane-based NAC from January 2012 to July 2017 were retrospectively analyzed. Clinical and pathological parameters of the patients were retrieved and the predictive factors for NAC response were evaluated.

Results 705 patients showed clinical response (cRes), and 131 patients acquired pathologic complete response (pCR). Patients with higher pretreatment Ki67 ( $\geq 14 \%$ ), tumor size $\geq 4 \mathrm{~cm}$, and positive clinical nodal had better clinical therapy response, while patients with negative ER and PR, higher pretreatment Ki67 ( $\geq 14 \%$ ), and tumor size $<4 \mathrm{~cm}$ were more probable to attain pCR. The pretreatment Ki67 could be used as a predictor of NAC only in luminal subtypes, and $25.5 \%$ were identified as an ideal cut-off point to differentiate the cRes from non-cRes cases. Although a decrease in Ki67 had been found in almost all molecular subtypes after NAC, no statistically significant differences were found in the decrease of Ki67 were validated between the cRes and non-cRes group in HER2-rich and triple-negative subtypes ( $P=0.488$ and $P=0.111$, respectively). Conclusions The best cut-off for pretreatment Ki67 in predicting the connection with the tumor size lessening was $25.5 \%$ in luminal subtype. Aggressive adjuvant systemic treatments should be considered for patients with HER2-rich and triplenegative subtype who exhibit tumor shrinkage in NAC but still have high levels of Ki67.
\end{abstract}

Keywords Breast cancer $\cdot$ Tumor response $\cdot$ Molecular subtypes $\cdot$ Neoadjuvant chemotherapy $\cdot$ Ki67

\section{Introduction}

Neoadjuvant chemotherapy (NAC) is a standard treatment for advanced breast cancer (BC) patients with the aim to decrease the extent of surgery [1]. Moreover, it is possible to evaluate the efficacy of NAC in a comparatively short

Electronic supplementary material The online version of this article (https://doi.org/10.1007/s10549-018-4730-1) contains supplementary material, which is available to authorized users.

Shengchun Liu

shengchuliu1968@126.com

1 Department of Breast Surgery, The First Affiliated Hospital of Chongqing Medical University, No.1 Youyi Road, Yuzhong District, Chongqing 400042, China time via therapeutic response, which lets tumor response to chemotherapeutic agents be monitored by this approach [2]. Based on the 2011 St Gallen consensus, there are four subtypes of BC: luminal, luminal-HER2, HER2-rich, and triple-negative on the basis of the immunohistochemistry results of ER, PR, and HER2 [3]. Many studies have revealed effective predictors of the response to NAC with different molecular subtypes [4-6], but some of these conclusions remain controversial.

ki67 level was related to tumor cell proliferation, which is the first immunohistochemical (IHC) marker that calls for a precise quantity [7]. A great number of studies have shown that Ki67 was regarded as the marker that provides prognosis for BC patients who have undergone NAC [8-10], and it has been observed that Ki67 is a factor that can predict the 
response to NAC $[7,11,12]$. However, despite the increasing evidence showing the predictive value according to molecular subtype, it is not clear whether Ki67 is identically helpful for predictive approaches in various subtypes, especially the changes of Ki67 during NAC.

In this clinical practice, we have evaluated whether pretreatment Ki67 levels are able to calculate the effectiveness of chemotherapy among molecular subtypes with the same chemotherapy regimen. Furthermore, we investigated whether the change of Ki67 between the needle biopsy and the residual tumor can be used as a predictor for NAC in different subtypes with a relatively large number cohort. In addition, the clinical and pathological response of NAC was also correlated to the conventional clinicopathological factors in this research.

\section{Methods}

\section{Patients and treatment}

Overall, 1062 consecutive patients with primary BC who were treated with both NAC and surgery at Breast Cancer Center of Chongqing at The First Affiliated Hospital of Chongqing Medical University from January 2012 to July
2017 were recruited in this retrospective research. Only patients who received $\geq 3$ courses of treatment with TEC were included, which included intravenous administration of cyclophosphamide $\left(500 \mathrm{mg} / \mathrm{m}^{2}\right)$, epirubicin $\left(75 \mathrm{mg} / \mathrm{m}^{2}\right)$, and docetaxel $\left(75 \mathrm{mg} / \mathrm{m}^{2}\right)$ each 21 days. Other exclusion criteria included bilateral $\mathrm{BC}$, male $\mathrm{BC}$, and a history of contralateral $\mathrm{BC}$, and finally 1010 patients were enrolled (Fig. 1). All HER-2 (+) patients were thoroughly informed of the effect of targeted therapy on the outcomes. However, only 3\% (11/356) HER-2 (+) patients accepted trastuzumab due to financial reasons. All 11 patients received 4 cycles of EC regimen, then followed by $\mathrm{T}+$ trastuzumab every 3 weeks (loading dose of $8 \mathrm{mg}$ per $\mathrm{kg}$ of bodyweight infused intravenously during $90 \mathrm{~min}$, followed by $6 \mathrm{mg} / \mathrm{kg}$ during 30 min every 3 weeks). After chemotherapy, they also received additional cycles of trastuzumab until 1 year. Furthermore, patients treated by trastuzumab monitored left ventricular ejection fraction (LVEF) at the beginning and end of chemotherapy, and none of them developed a heart complication (the absolute drops of LVEF were $0-7 \%)$.

This research was authorized by the ethics committee of The First Affiliated Hospital of Chongqing Medical University. All patients received and agreed with the informed consent.
Fig. 1 Chemo-effects and patient registration. Included and excluded in the analysis according to the availability of tumor material and treatment group ( $E$ epirubicin, $T$ docetaxel, $C$ cyclophosphamide, $F$ fluorouracil, $N$ vinorelbine, $P$ Cisplatin)

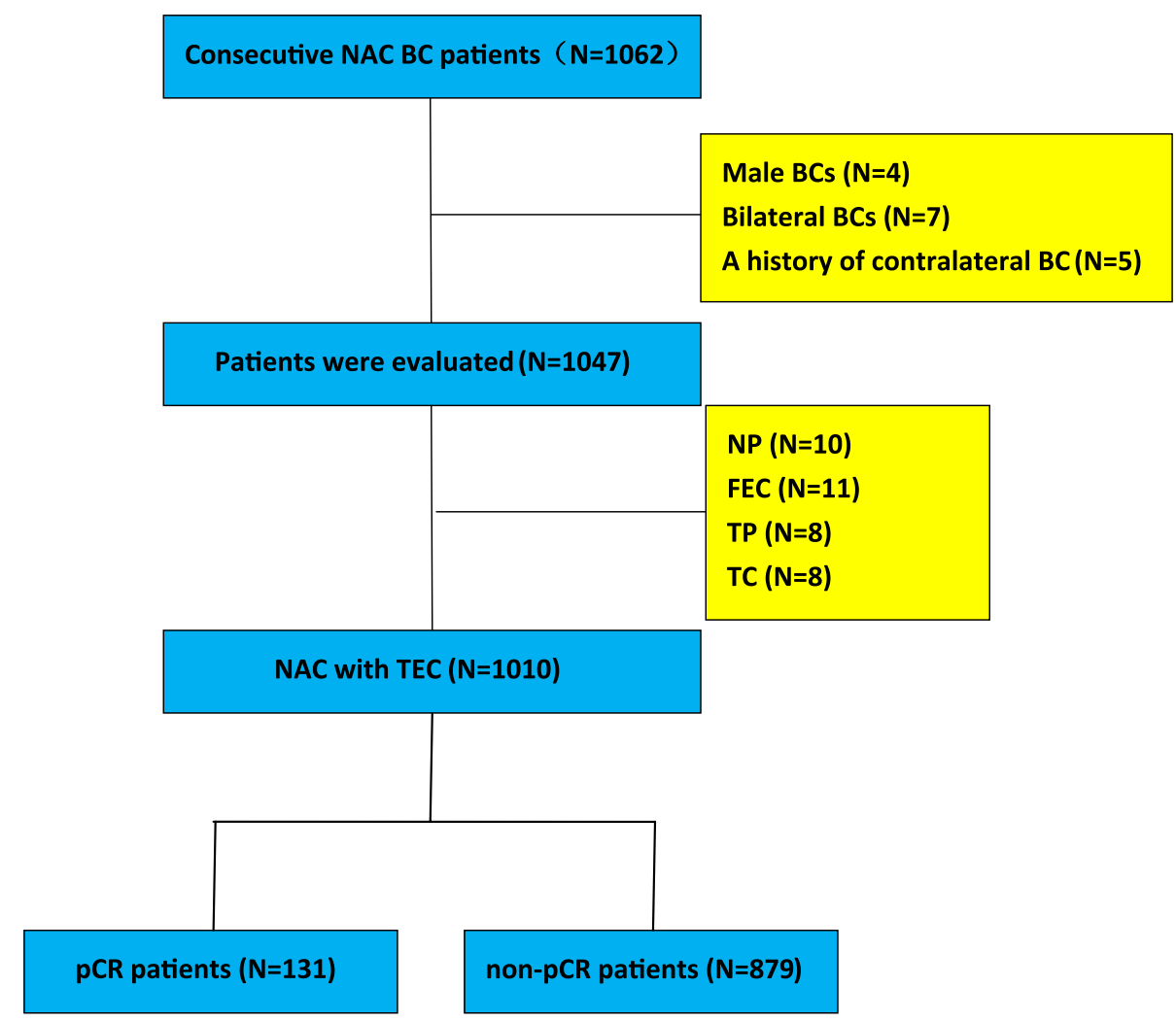




\section{Immunohistochemical staining and intrinsic subtypes}

ER, PR, HER-2 status, and Ki-67 index were measured before and after NAC by IHC. All results were evaluated independently by two pathologists. If more than $1 \%$ of nuclei were colored, we considered that ER and PR were positive [13]. If the specimen either recorded $3+$ by IHC, or demonstrated an over 2.2-fold growth in fluorescence in situ hybridization (FISH), it could be regarded as HER2-positive (Fig. 1S). Tumor subtypes were defined based on the expression of ER, PR, and HER2: luminal (ER+ and/or PR+, HER2-), luminal-HER2 (ER+ and/or PR+, HER2+), HER2-rich $(\mathrm{ER}-$ and $\mathrm{PR}-, \mathrm{HER} 2+)$, and triple negative (ER - and PR- and HER2-). The Ki-67 value was explained as the proportion of positive cells (500-1000) with nuclear staining in the invasive front of the tumor as advised by the International Ki67 in Breast Cancer Working Group [14].

\section{Evaluation of the response to chemotherapy}

Clinical diagnostic imaging (ultrasonography and magnetic resonance imaging) was utilized to measure the reaction of $\mathrm{BC}$ during NAC. Clinical response was specified by making the comparison of the alteration of primary lesions. Physical and imaging examinations based on Response Evaluation Criteria in Solid Tumors (RECIST) guidelines version 1.1 were utilized to assess treatment response as follows: cPR reduction in a total of target lesion diameters $\geq 30 \%$; cPD growth in a total of target lesion diameters $\geq 20 \%$; cSD neither sufficient reduction as cPR nor sufficient growth as cPD. Pathologic complete response (pCR) was explained as no remaining invasive disease in any excised breast tissue irrespective of nodal involvement [9].

\section{Statistical analysis}

The comparison among quantitative characteristics was made by Kruskal-Wallis test, and the comparison among categorical features was displayed by $\chi^{2}$ test. Receiver operating characteristic (ROC) curve analysis was employed to measure the cutoff value of Ki67 indication. The whole of the analyses was conducted through SPSS version 22.0 (SPSS Inc, Chicago, USA). In this study, $P$ value less than 0.05 was regarded as significant in statistical respect and every $P$ value was two-sided.

\section{Results}

\section{Baseline characteristics}

One thousand and ten females with primary BC were inclusive. Chemotherapy was organized for a median of
Table 1 Clinicopathological features of the study cohort $(\mathrm{n}=1010)$

\begin{tabular}{|c|c|}
\hline Parameters & Number $(\%)$ \\
\hline \multicolumn{2}{|l|}{ Age (year) } \\
\hline$<40$ & $133(13.2)$ \\
\hline$\geq 40$ & $877(86.8)$ \\
\hline \multicolumn{2}{|l|}{ Menopause } \\
\hline Yes & $399(39.5)$ \\
\hline No & $611(60.5)$ \\
\hline \multicolumn{2}{|l|}{ Chemotherapy cycles } \\
\hline 3 & $29(2.9)$ \\
\hline 4 & $900(89.2)$ \\
\hline $5-8$ & $81(7.9)$ \\
\hline \multicolumn{2}{|l|}{ Subtypes of cancer } \\
\hline Ductal & $986(97.6)$ \\
\hline Lobular & $13(1.3)$ \\
\hline Others & $11(1.1)$ \\
\hline \multicolumn{2}{|l|}{ Tumor size } \\
\hline$<2 \mathrm{~cm}$ & $74(7.3)$ \\
\hline $2-4 \mathrm{~cm}$ & $522(51.7)$ \\
\hline$\geq 4 \mathrm{~cm}$ & $414(41.0)$ \\
\hline \multicolumn{2}{|l|}{ Clinical nodal status } \\
\hline Positive & $473(46.8)$ \\
\hline Negative & $537(53.2)$ \\
\hline \multicolumn{2}{|l|}{ Histological grade } \\
\hline I & $26(2.6)$ \\
\hline II & $578(57.3)$ \\
\hline III & $130(12.9)$ \\
\hline Unknown & $276(27.2)$ \\
\hline \multicolumn{2}{|l|}{ Response evaluation } \\
\hline $\mathrm{pCR}$ & $131(13.0)$ \\
\hline $\mathrm{cPR}$ & $574(56.8)$ \\
\hline $\mathrm{cSD}$ & $290(28.7)$ \\
\hline cPD & $15(1.5)$ \\
\hline Responder (pCR and cPR) & $705(69.8)$ \\
\hline Non-responder (cSD and cPD) & $305(30.2)$ \\
\hline
\end{tabular}

$p C R$ pathological complete response, $c P R$ clinical partial response, $c P D$ clinical progressive disease, $c S D$ clinical stable

4 cycles (range $3-8$ cycles) before operation. The median age of the enrolled patients was $49 \pm 8.485$ years (range 20-72 years), and $39.5 \%$ of them were menopausal. The mean tumor size before and after NAC were $4.0 \pm 3.89$ and $2.12 \pm 1.98 \mathrm{~cm}$, respectively, and $41.0 \%$ of them had node-positive disease at diagnosis. One hundred and thirty-one patients $(13.0 \%)$ achieved pCR, and 574 patients (56.8\%) showed clinical response to NAC based on the RECIST criteria, while 305 patients $(30.4 \%)$ showed no response, including 290 of $\mathrm{cSD}(28.7 \%)$ and 15 of $\mathrm{cPD}$ $(1.5 \%)$. Patients, tumor, and treatment baseline characteristics as well as tumor reaction are detailed in Table 1. 
$66.3 \%$ of the whole cases showed ER-positive (31 cases with $1-10 \%$ positive) and $52.8 \%$ of the examined samples demonstrated PR positivity (20 cases with $1-10 \%$ positive). $35.2 \%$ of cases provided the evidence of HER 2 positivity, and $70.8 \%$ of all patients had the expression of $\mathrm{Ki} 67 \geq 14 \%$. Within this study, $36.8 \%(n=372)$ were categorized as luminal type, $16.8 \%(n=170)$ as luminal-HER2, $18.4 \%$ $(n=186)$ as HER2-rich, and $14.1 \%(n=142)$ as triple negative. The Immunochemical data and molecular subtype features are shown in Table 2.

\section{Association between baseline characteristics and clinical or pathological response to NAC}

Chi-square test $\left(\chi^{2}\right)$ was utilized to assess the relationship between the clinical pathological parameters and the clinical or pathological response to NAC. As shown in Table 3, Age and HER2 status did not demonstrate a significant response to NAC in statistical results (all $P>0.05$ ). Patients with greater Ki67 level ( $\geq 14 \%)$ had better clinical and pathological response to NAC $(P<0.001)$. In the clinical response assessment, patients with positive ER and PR status did

Table 2 Immunochemical data and molecular subtype features of the cohort $(n=1010)$

\begin{tabular}{lc}
\hline Parameters & Number $(\%)$ \\
\hline ER positivity score & \\
0 & $340(33.7)$ \\
$0-10 \%$ & $31(3.1)$ \\
$\geq 10 \%$ & $639(63.2)$ \\
PR positivity score ${ }^{\mathrm{a}}$ & \\
0 & $477(47.2)$ \\
$0-10 \%$ & $20(2.0)$ \\
$\geq 10 \%$ & $513(50.8)$ \\
Her-2 & \\
Positive & $356(35.2)$ \\
Negative & $514(50.9)$ \\
Unknown & $140(13.9)$ \\
Ki67 & \\
$<14 \%$ & $295(29.2)$ \\
$\geq 14 \%$ & $715(70.8)$ \\
Molecular subtype & \\
Luminal & $372(36.8)$ \\
Luminal/HER2 & $170(16.8)$ \\
HER2 & $186(18.4)$ \\
TN & $142(14.1)$ \\
Unknown & $140(13.9)$ \\
\hline
\end{tabular}

$E R$ estrogen receptor, $P R$ progesterone receptor, HER2 human epidermal growth factor receptor 2

${ }^{a}$ Positive $\geq 1 \%$

bLuminal: HR+/HER2-, luminal/HER2: HR+/HER2+, HER2: HR-/HER2+, TN: HR-/HER2- not show significance in statistical respect $(P=0.904$ and $P=0.542$, separately) while the tumor size $(\geq 4 \mathrm{~cm})$ and positive clinical nodal were significantly associated with tumor size reduction $(P<0.001$ and $P=0.03$, respectively), and the menstrual status had marginal $\mathrm{P}$ values $(\mathrm{P}=0.058)$. In the pathological response assessment, patients with negative ER and PR status were more likely to achieve pCR (both $P<0.001)$. Furthermore, the tumor diameter $(<4 \mathrm{~cm})$ was significantly connected with pCR $(\mathrm{P}=0.038)$, whereas the menstrual and clinical nodal status did not show statistical significance $(P=0.962$ and $P=0.289$, separately).

In addition, the response to NAC between patients with weakly hormone receptor (HR) (1-10\% positive) and $\mathrm{HR} \geq 10 \%$ or HR - were also assessed. As shown in Fig. S2, patients with HR 1-10\% positive did not demonstrate a significant response to NAC in statistical results compared with $\mathrm{HR} \geq 10 \%$ or HR - patients (all $P>0.05$ ).

\section{Evaluation of the predictive value of pretreatment and decreased Ki67 during NAC in different molecular subtypes}

ROC curve analysis was utilized to identify the predictive value of pretreatment Ki67 expression response to NAC in different molecular subtypes (Fig. 2). Area under ROC curve (AUC) of Ki67 expression was 0.632 in luminal-type BCs $(P<0.001,95 \%$ CI 0.565-0.686). On the contrary, the AUC of Ki67 expression were 0.508, 0.548, and 0.54 in luminal-HER2, HER2-rich, and triple-negative type BCs separately, demonstrating that Ki67 level according to biopsy specimen was ineffective in forecasting of therapeutic response among these subtypes $(P=0.869, P=0.303$, and $P=0.448$, respectively). Accordingly, the result of ROC curve analysis showed that pretreatment Ki67 expression could not be used as a predictor of NAC in luminal/HER2 subtypes with HR $1-10 \%$ positive $(P=0.357$, Fig. 3S). Furthermore, in luminal-type $\mathrm{BCs}$, we recognized that $25.5 \%$ as the best cut-off value of pretreatment Ki67 for predicting response to NAC with an ideal sensitivity of $46.6 \%$ and a specificity of $69.9 \%$. On the other hand, the positive predictive value and negative predictive value were 78.5 and $39.2 \%$, respectively.

To assess the changes in Ki67 during NAC, differences in Ki67 expression between biopsy and surgical specimens from the same patient were evaluated in 879 patients. As shown in Fig. 3, post-NAC Ki67 levels were greatly reduced in luminal, luminal-HER2, and HER2-rich subtypes ( $P<0.001, P<0.001$, and $P=0.01$, separately $)$, and the triple-negative type BCs had marginal $P$ values $(P=0.061)$. We also explored the association between tumor size reduction and the decrease of Ki67 in different molecular subtypes (Fig. 4). In our analysis, tumor size reduction was closely related to the decrease of Ki67 during NAC in luminal and 
Table 3 Clinicopathological characteristics of pre-NAC according to clinical and pathological response

\begin{tabular}{|c|c|c|c|c|c|c|}
\hline \multirow[t]{2}{*}{ Characteristic } & \multicolumn{3}{|c|}{ Clinical response } & \multicolumn{3}{|c|}{ Pathology response } \\
\hline & cRes & Non-cRes & $P$ value & $\mathrm{pCR}$ & Non-pCR & $P$ value \\
\hline Age (year) & & & 0.384 & & & 0.466 \\
\hline$<40$ & 95 & 35 & & 20 & 113 & \\
\hline$\geq 40$ & 610 & 270 & & 111 & 766 & \\
\hline Menopause & & & 0.058 & & & 0.962 \\
\hline Yes & 265 & 134 & & 52 & 347 & \\
\hline No & 440 & 171 & & 79 & 532 & \\
\hline Tumor size (cm) & & & $<0.001$ & & & 0.039 \\
\hline$<4$ & 391 & 205 & & 88 & 505 & \\
\hline$\geq 4$ & 314 & 100 & & 43 & 371 & \\
\hline Clinical nodal status & & & 0.03 & & & 0.289 \\
\hline Yes & 346 & 127 & & 67 & 406 & \\
\hline No & 359 & 178 & & 64 & 473 & \\
\hline ER status & & & 0.904 & & & $<0.001$ \\
\hline Positive & 442 & 190 & & 56 & 576 & \\
\hline Negative & 263 & 115 & & 75 & 303 & \\
\hline PR status & & & 0.542 & & & $<0.001$ \\
\hline Positive & 332 & 150 & & 32 & 450 & \\
\hline Negative & 373 & 155 & & 99 & 429 & \\
\hline Her2 status & & & 0.092 & & 318 & 0.233 \\
\hline Positive & 270 & 96 & & 48 & & \\
\hline Negative & 348 & 166 & & 54 & 460 & \\
\hline Ki67 expression (\%) & & & $<0.001$ & & & $<0.001$ \\
\hline$<14$ & 182 & 113 & & 16 & 279 & \\
\hline$\geq 14$ & 523 & 192 & & 115 & 600 & \\
\hline
\end{tabular}

cRes $\mathrm{pCR}$ and $\mathrm{cPR}$, Non-cRes $\mathrm{cPD}$ and $\mathrm{cSD}$
luminal-HER2 subtypes $(P<0.001$ and $P=0.048$, separately). On the contrary, Insignificant statistical distinctions in the decrease of Ki67 during NAC were identified between the cRes and non-cRes group in HER2-rich and triple-negative subtypes ( $P=0.488$ and $P=0.111$, separately).

\section{Discussion}

NAC, which downstages the disease and reduces tumor volume, has been resulted in improved success rates of breastconserving operations. Furthermore, the patients who attain pCR after NAC have a very low risk of relapse and death regardless of the earlier stage and molecular subtype $[4,15]$. In the latest years, it has been accepted that $\mathrm{BC}$ can be categorized into multiple subtypes by IHC analysis of ER, PR, HER-2, and Ki67, and several studies revealed the response to NAC in different molecular subtypes $[1,3,5,6]$. However, some of these conclusions remain controversial $[11,16]$.

A great number of studies have demonstrated a positive connection between Ki67 expression and chemotherapy response $[2,8,17,18]$. In some studies, Ki67 is thought to predict NAC response only in ER-positive BCs [2, 18-20].
However, some clinical trial with relatively large cases suggested that Ki67 independently improved the prediction of treatment response in luminal tumors as well as triple-negative tumors $[8,17,21]$. Furthermore, although some studies revealed that Ki67 changes play different predictive roles in ER-positive and ER-negative patients during NAC, the limited number of cases limits further typing analysis [12, 22].

In this study, we first assessed the usefulness of commonly applied tumor characteristics to forecast clinical response and pCR after NAC. In the clinical response assessment, pretreatment Ki67 ( $\geq 14 \%$ ), tumor size $\geq 4 \mathrm{~cm}$, and positive clinical nodal were significantly associated with tumor size reduction, whereas Age, ER, PR, and HER2 status did not show a statistically significant response to NAC. In addition, the menstrual status had marginal $P$ values in this analysis. Furthermore, patients with negative ER, PR, and smaller tumor size $(<4 \mathrm{~cm})$ were more probable to attain pCR, while the Age, HER2 status, menstrual, and clinical nodal status did not show statistically significant response in pathological response assessment. Moreover, the response to NAC of patients with HR 1-10\% positive were also evaluated because recent research indicated that weakly HR expression was a poor correlate of luminal subtype [23], 

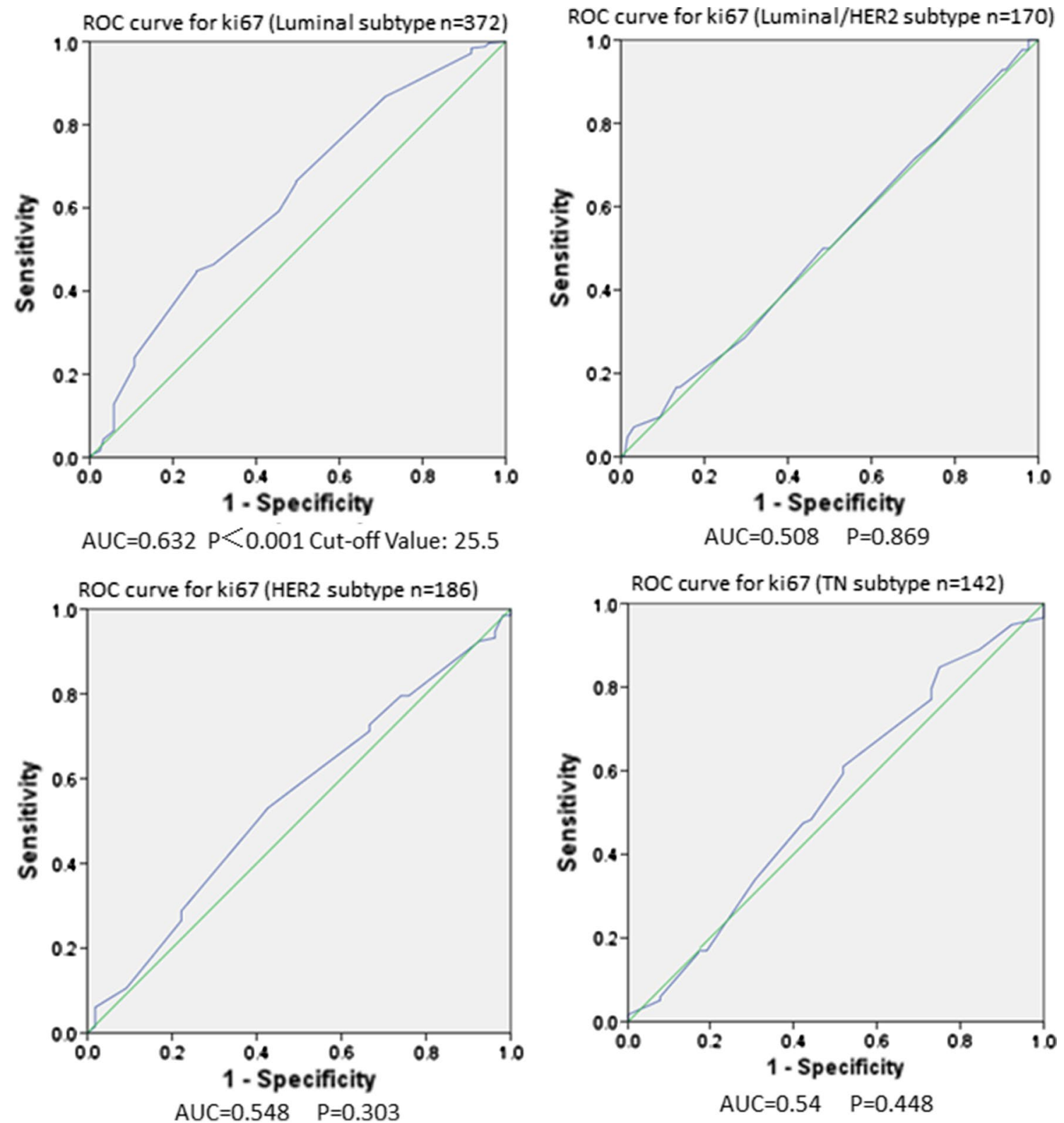

Fig. 2 Comparison of the predictive role of pre-NAC Ki67 for the response to NAC by ROC curve analysis among the different molecular subtypes

and the therapeutic effect of hormone therapy of patients with HR 1-10\% positive was poor [24]. According to our results, patients with HR 1-10\% positive did not demonstrate a significant response to NAC in statistics compared with $\mathrm{HR} \geq 10 \%$ or HR- patients. However, the number of patients with HR $1-10 \%$ positive in our study was relatively small $(n=31)$, so it is necessary to further confirm the above relationship through large sample studies.

Most of the above results were in line with previous literature $[12,16,19,25]$. However, the HER2 status did not show a statistically significant response in clinical and pathological response in our analysis, which is not consistent with the existing literature. The possible explanation is that only 3\% HER $2(+)$ patients accept trastuzumab before surgery in this study, while this phenomenon also confirms the importance of using trastuzumab in HER2 $(+)$ patients during NAC [26, 27].

The overall pCR rate in our study was $13 \%$, which was relatively low compared with some large studies (15.8-27.1\%) $[9,28,29]$. We speculated that this phenomenon may be due to relatively short cycles of NAC in this study $(89.2 \%$ of patients experienced 4 cycles of chemotherapy) compared with the 8 cycles of NAC in other studies [9, 29]. Another possible reason for this is that the proportion of patients with 

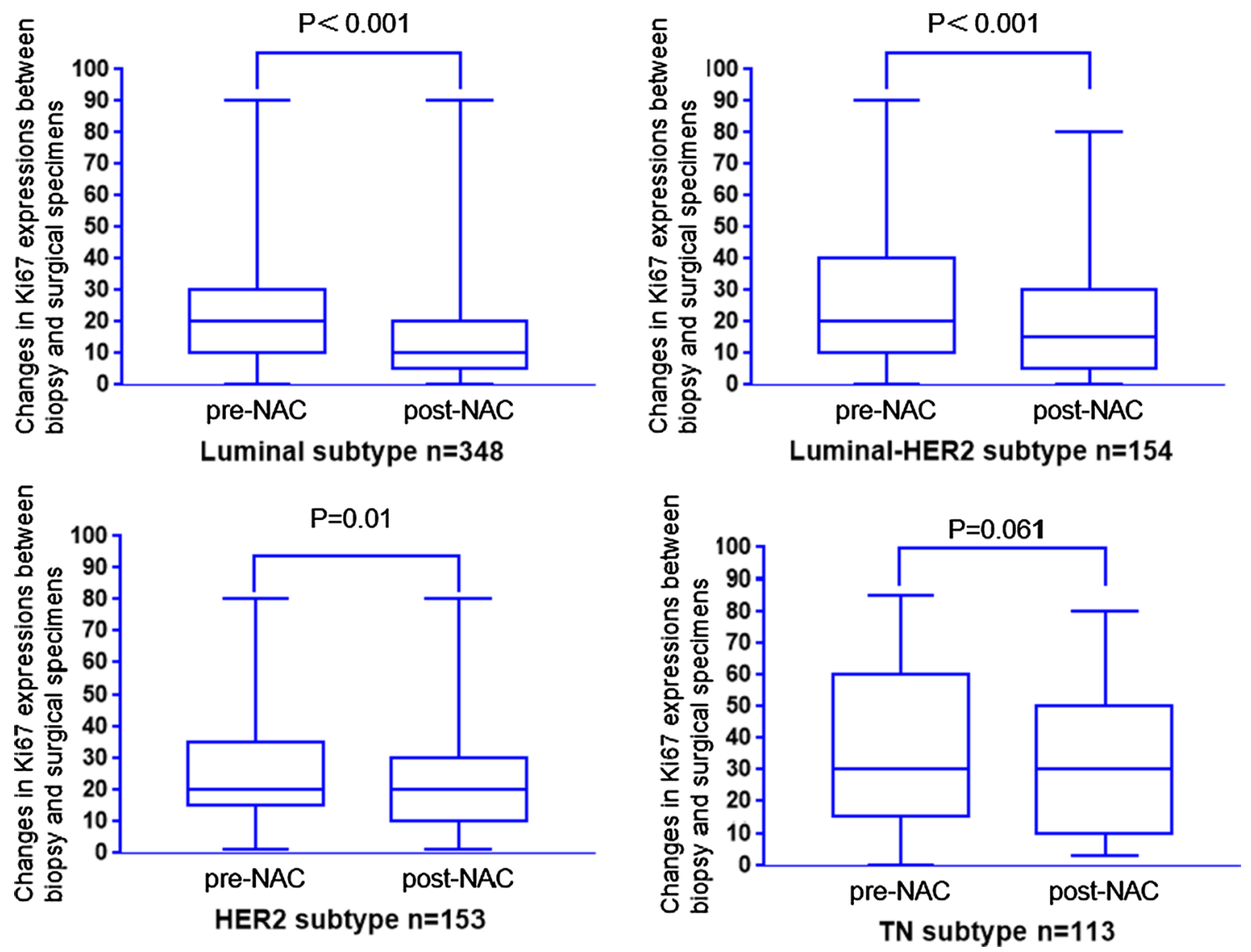

Fig. 3 Changes in Ki67 expression between biopsy and surgical specimens in the different molecular subtypes

clinical tumor size $\geq 4 \mathrm{~cm}$ in our study reached $41 \%$, which was significantly higher than the $28 \%$ of NSABP B- 27 trial [15] because NAC may aid in shrinking the size of the tumor instead of directly leading to pCR status [30].

Next, we focused on evaluating the predictive role of Ki67 expression in different molecular subtypes for response to NAC. According to our findings, the pretreatment Ki67 could be used as a predictor of NAC only in luminal-type BCs. With a ROC curve, we identified $25.5 \%$ as the best cut-off value of pretreatment Ki67 for predicting response to NAC with an optimal sensitivity of $46.6 \%$ and a specificity of $69.9 \%$. To assess the impact of Ki67 changes on response to treatment, we first investigated the changes in Ki67 after NAC in different molecular subtypes. As expected, a decrease in Ki67 had been found in almost all molecular subtypes after NAC (the triple-negative type BCs had a borderline significant), which was consistent with previous research [18]. However, when considering the distribution of responders and non-responders, the reduction in Ki67 may induce a positive response only in luminal and luminal-HER2 subtypes.

Our results show that Ki67 has been demonstrated to forecast NAC response only in ER-positive BCs, which are consistent with several studies [2, 12, 17, 18, 20]. Furthermore, our research also revealed that the reduction in Ki67 could not be used as a predictor of NAC in HER2-rich and triple-negative subtypes, which was in line with previous study [12]. It has been shown that the post-treatment, but not pretreatment Ki67 indication levels identify a group of patients at great danger for relapse in patients who do not achieve pCR [9]. Therefore, patients with HER2-rich and triple-negative subtype who exhibit marked tumor shrinkage in NAC but still have high levels of Ki67 might be good candidates for more aggressive adjuvant systemic treatments, such as extension of postoperative chemotherapy, proper radiotherapy, and administration of Capecitabine, which was recently shown to prolong the survival of nonpCR patients [31]. Another interesting finding in this study is that the response to NAC in patients with luminal-HER2 subtype can be predicted by the reduction in Ki67, but not by pretreatment Ki67. Possible explanations for this phenomenon are tumor heterogeneity [32], different signaling pathways caused by chemotherapy [33], and the presence of more molecular subtypes.

The major limitation of this study is that our data come from a retrospective study in one single center. Therefore, it 

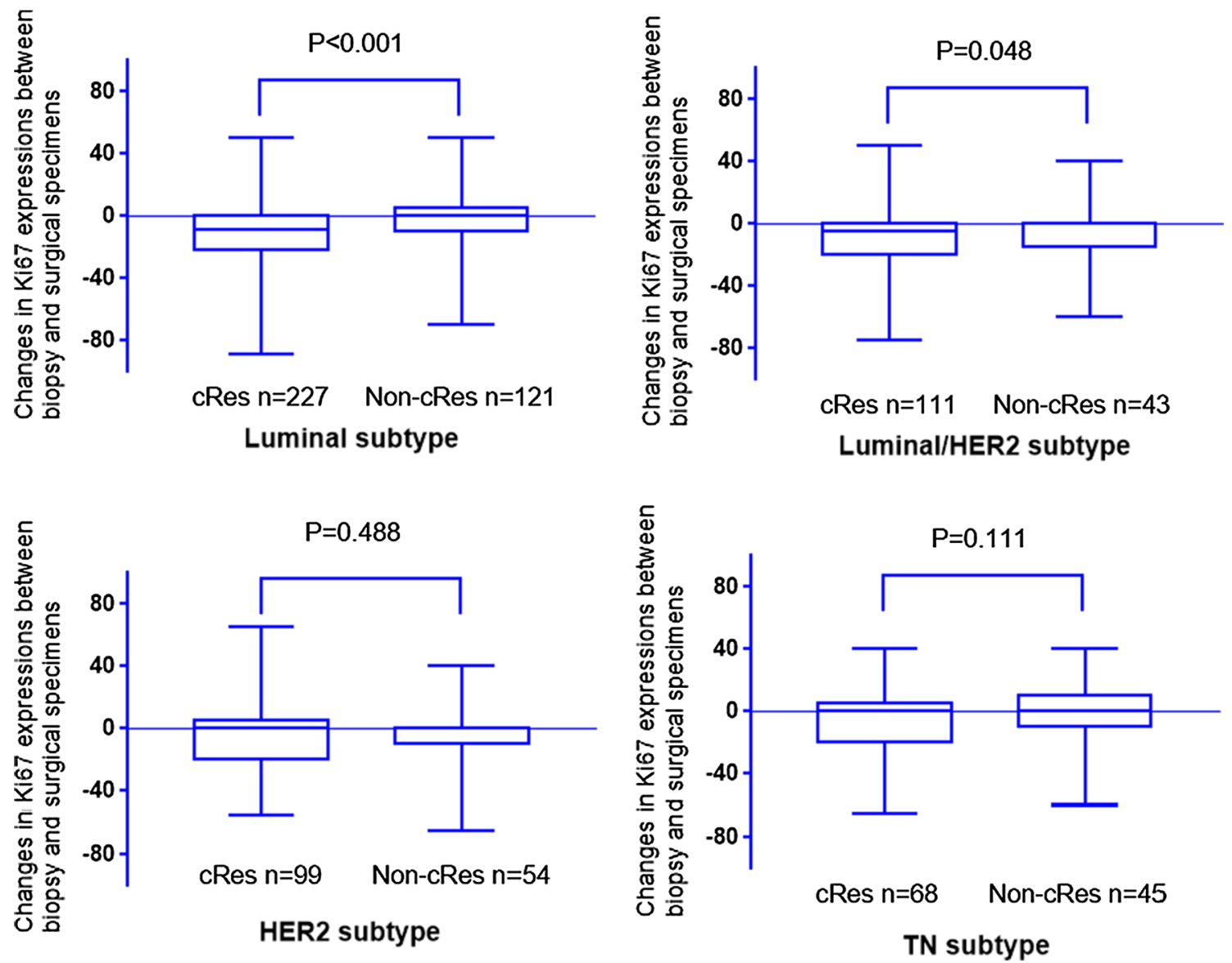

Fig. 4 Changes in Ki67 expression between biopsy and surgical specimens according to patient outcomes among the different molecular subtypes

is necessary to perform prospective study to obtain evidence supporting our results.

\section{Conclusion}

This analysis suggested that the pretreatment and decreased Ki67 play different predictive roles in different molecular subtypes treated with NAC. The best cut-off for pretreatment Ki67 in predicting the connection with the tumor size decrease was $25.5 \%$ in luminal subtype. In addition, aggressive adjuvant systemic treatments should be considered for patients with HER2-rich and triple-negative subtype who exhibit tumor shrinkage in NAC but still have high levels of $\mathrm{Ki} 67$.

Acknowledgements This study was supported by the National Natural Science Foundation of china (NSFC: no.81472658) and Chongqing science and technology committee fund (csct2015shmszx0269).
Open Access This article is distributed under the terms of the Creative Commons Attribution 4.0 International License (http://creativeco mmons.org/licenses/by/4.0/), which permits unrestricted use, distribution, and reproduction in any medium, provided you give appropriate credit to the original author(s) and the source, provide a link to the Creative Commons license, and indicate if changes were made.

\section{References}

1. Kaufmann M, von Minckwitz G, Mamounas EP, Cameron D, Carey LA, Cristofanilli M et al (2012) Recommendations from an international consensus conference on the current status and future of neoadjuvant systemic therapy in primary breast cancer. Ann Surg Oncol 19(5):1508-1516

2. Kim KI, Lee KH, Kim TR, Chun YS, Lee TH, Park HK (2014) $\mathrm{Ki}-67$ as a predictor of response to neoadjuvant chemotherapy in breast cancer patients. J Breast Cancer 17(1):40-46

3. Vasconcelos I, Hussainzada A, Berger S, Fietze E, Linke J, Siedentopf F et al (2016) The St. Gallen surrogate classification for breast cancer subtypes successfully predicts tumor presenting features, nodal involvement, recurrence patterns and disease free survival. Breast 29:181-185 
4. Schott AF, Hayes DF (2012) Defining the benefits of neoadjuvant chemotherapy for breast cancer. J Clin Oncol 30(15):1747-1749

5. Millar EK, Graham PH, O'Toole SA, McNeil CM, Browne L, Morey AL et al (2009) Prediction of local recurrence, distant metastases, and death after breast-conserving therapy in earlystage invasive breast cancer using a five-biomarker panel. J Clin Oncol 27(28):4701-4708

6. Symmans WF, Wei C, Gould R, Yu X, Zhang Y, Liu M et al (2017) Long-term prognostic risk after neoadjuvant chemotherapy associated with residual cancer burden and breast cancer subtype. J Clin Oncol 35(10):1049-1060

7. Polley MY, Leung SC, McShane LM, Gao D, Hugh JC, Mastropasqua MG et al (2013) An international Ki67 reproducibility study. J Natl Cancer Inst 105(24):1897-1906

8. Denkert C, Loibl S, Muller BM, Eidtmann H, Schmitt WD, Eiermann W et al (2013) Ki67 levels as predictive and prognostic parameters in pretherapeutic breast cancer core biopsies: a translational investigation in the neoadjuvant GeparTrio trial. Ann Oncol 24(11):2786-2793

9. von Minckwitz G, Schmitt WD, Loibl S, Muller BM, Blohmer JU, Sinn BV et al (2013) Ki67 measured after neoadjuvant chemotherapy for primary breast cancer. Clin Cancer Res 19(16):4521-4531

10. Luporsi E, Andre F, Spyratos F, Martin PM, Jacquemier J, Penault-Llorca F et al (2012) Ki-67: level of evidence and methodological considerations for its role in the clinical management of breast cancer: analytical and critical review. Breast Cancer Res Treat 132(3):895-915

11. Andre F, Arnedos M, Goubar A, Ghouadni A, Delaloge S (2015) Ki67-no evidence for its use in node-positive breast cancer. Nat Rev Clin Oncol 12(5):296-301

12. Enomoto Y, Morimoto T, Nishimukai A, Higuchi T, Yanai A, Miyagawa $\mathrm{Y}$ et al (2016) Impact of biomarker changes during neoadjuvant chemotherapy for clinical response in patients with residual breast cancers. Int J Clin Oncol 21(2):254-261

13. Hammond ME, Hayes DF, Dowsett M, Allred DC, Hagerty KL, Badve $S$ et al (2010) American Society of Clinical Oncology/ College of American Pathologists guideline recommendations for immunohistochemical testing of estrogen and progesterone receptors in breast cancer (unabridged version). Arch Pathol Lab Med 134(7):e48-e72

14. Dowsett M, Nielsen TO, A'Hern R, Bartlett J, Coombes RC, Cuzick J et al (2011) Assessment of Ki67 in breast cancer: recommendations from the International Ki67 in Breast Cancer working group. J Natl Cancer Inst 103(22):1656-1664

15. Rastogi P, Anderson SJ, Bear HD, Geyer CE, Kahlenberg MS, Robidoux A et al (2008) Preoperative chemotherapy: updates of National Surgical Adjuvant Breast and Bowel Project Protocols B-18 and B-27. J Clin Oncol 26(5):778-785

16. Brouckaert O, Paridaens R, Floris G, Rakha E, Osborne K, Neven P (2013) A critical review why assessment of steroid hormone receptors in breast cancer should be quantitative. Ann Oncol 24(1):47-53

17. Fasching PA, Heusinger K, Haeberle L, Niklos M, Hein A, Bayer $\mathrm{CM}$ et al (2011) Ki67, chemotherapy response, and prognosis in breast cancer patients receiving neoadjuvant treatment. BMC Cancer 11:486

18. Matsubara N, Mukai H, Fujii S, Wada N (2013) Different prognostic significance of $\mathrm{Ki}-67$ change between pre- and post-neoadjuvant chemotherapy in various subtypes of breast cancer. Breast Cancer Res Treat 137(1):203-212

19. Jones RL, Salter J, A'Hern R, Nerurkar A, Parton M, Reis-Filho JS et al (2010) Relationship between oestrogen receptor status and proliferation in predicting response and long-term outcome to neoadjuvant chemotherapy for breast cancer. Breast Cancer Res Treat 119(2):315-323

20. Acs B, Zambo V, Vizkeleti L, Szasz AM, Madaras L, Szentmartoni $\mathrm{G}$ et al (2017) Ki-67 as a controversial predictive and prognostic marker in breast cancer patients treated with neoadjuvant chemotherapy. Diagn Pathol 12(1):20

21. Munzone E, Botteri E, Sciandivasci A, Curigliano G, Nole F, Mastropasqua $\mathrm{M}$ et al (2012) Prognostic value of Ki-67 labeling index in patients with node-negative, triple-negative breast cancer. Breast Cancer Res Treat 134(1):277-282

22. Tokuda E, Horimoto Y, Arakawa A, Himuro T, Senuma K, Nakai $\mathrm{K}$ et al (2017) Differences in Ki67 expressions between pre- and post-neoadjuvant chemotherapy specimens might predict early recurrence of breast cancer. Hum Pathol 63:40-45

23. Sheffield BS, Kos Z, Asleh-Aburaya K, Wang XQ, Leung S, Gao $D$ et al (2016) Molecular subtype profiling of invasive breast cancers weakly positive for estrogen receptor. Breast Cancer Res Treat 155(3):483-490

24. Iwamoto T, Booser D, Valero V, Murray JL, Koenig K, Esteva FJ et al (2012) Estrogen receptor (ER) mRNA and ER-related gene expression in breast cancers that are 1-10\% ER-positive by immunohistochemistry. J Clin Oncol 30(7):729-734

25. Gomez R, Ossa CA, Montoya ME, Echeverri C, Angel G, Ascuntar J et al (2015) Impact of immunohistochemistry-based molecular subtype on chemosensitivity and survival in Hispanic breast cancer patients following neoadjuvant chemotherapy. Ecancermedicalscience 9:562

26. Gianni L, Eiermann W, Semiglazov V, Manikhas A, Lluch A, Tjulandin $S$ et al (2010) Neoadjuvant chemotherapy with trastuzumab followed by adjuvant trastuzumab versus neoadjuvant chemotherapy alone, in patients with HER2-positive locally advanced breast cancer (the NOAH trial): a randomised controlled superiority trial with a parallel HER2-negative cohort. Lancet 375(9712):377-384

27. Slamon D, Eiermann W, Robert N, Pienkowski T, Martin M, Press $M$ et al (2011) Adjuvant trastuzumab in HER2-positive breast cancer. N Engl J Med 365(14):1273-1283

28. Klauschen F, Wienert S, Schmitt WD, Loibl S, Gerber B, Blohmer JU et al (2015) Standardized Ki67 diagnostics using automated scoring-clinical validation in the gepartrio breast cancer study. Clin Cancer Res 21(16):3651-3657

29. Bear HD, Anderson S, Brown A, Smith R, Mamounas EP, Fisher $B$ et al (2003) The effect on tumor response of adding sequential preoperative docetaxel to preoperative doxorubicin and cyclophosphamide: preliminary results from National Surgical Adjuvant Breast and Bowel Project Protocol B-27. J Clin Oncol 21(22):4165-4174

30. Lim LY, Miao H, Lim JS, Lee SC, Bhoo-Pathy N, Yip CH et al (2017) Outcome after neoadjuvant chemotherapy in Asian breast cancer patients. Cancer Med 6(1):173-185

31. Masuda N, Lee SJ, Ohtani S, Im YH, Lee ES, Yokota I et al (2017) Adjuvant capecitabine for breast cancer after preoperative chemotherapy. N Engl J Med 376(22):2147-2159

32. Yuan Y, Failmezger H, Rueda OM, Ali HR, Graf S, Chin SF et al (2012) Quantitative image analysis of cellular heterogeneity in breast tumors complements genomic profiling. Sci Transl Med. 4(157):157ra43

33. Lewis-Wambi JS, Jordan VC (2009) Estrogen regulation of apoptosis: how can one hormone stimulate and inhibit? Breast Cancer Res 11(3):206 